\title{
Collider probes of singlet fermionic dark matter scenarios for the Fermi gamma-ray excess
}

\author{
Yeong Gyun Kim, ${ }^{a}$ Chan Beom Park ${ }^{b, 1}$ and Seodong Shin ${ }^{c, d, 1}$ \\ ${ }^{a}$ Department of Science Education, Gwangju National University of Education, \\ 55 Pilmundaero, Buk-gu, Gwangju 61204, Korea \\ ${ }^{b}$ Center for Theoretical Physics of the Universe, Institute for Basic Science (IBS), \\ 55 Expo-ro, Yuseong-gu, Daejeon 34051, Korea \\ ${ }^{c}$ Enrico Fermi Institute, University of Chicago, \\ 933 East 56th Street, Chicago, IL 60637, U.S.A. \\ ${ }^{d}$ Department of Physics and IPAP, Yonsei University, \\ 50 Yonsei-ro, Seodaemun-gu, Seoul 03722, Korea \\ E-mail: ygkim@gnue.ac.kr, cbpark@ibs.re.kr, seodongshin@yonsei.ac.kr
}

ABSTRACT: We investigate the collider signatures of three benchmark points in the singlet fermionic dark matter model motivated by the Fermi gamma-ray excess at the Galactic center. The benchmark points are classified according to the final state of the main dark annihilation process: a pair of $b$ quarks, Higgs bosons and new scalar bosons. Since the dark sector in the model communicates with the visible sector through a mixing between the new scalar and the Higgs boson, collider observables related to the measurements of Higgs boson properties and searches for the new scalar boson are essential to probe the benchmark scenarios at colliders. In this paper, we consider four collider observables: (1) Higgs signal strength (essentially $h Z Z$ coupling), (2) triple Higgs coupling, (3) exotic Higgs decay, and (4) direct production of the new scalar particle. We find that the benchmark points have distinctive collider signatures that can be well tested by the interplay of future lepton and hadron colliders.

KeYwords: Beyond Standard Model, Higgs Physics

ARXIV EPRINT: 1809.01143

\footnotetext{
${ }^{1}$ Corresponding author.
} 


\section{Contents}

1 Introduction 1

2 Singlet fermionic dark matter 2

3 Benchmark points and collider signatures 4

3.1 BP I $(\psi \bar{\psi} \rightarrow b \bar{b}$ annihilation channel $) \quad 5$

3.1.1 Higgs signal strength 5

3.1.2 Triple Higgs coupling 6

3.1.3 Exotic Higgs decays 6

3.1.4 Production of $h_{2}$ at colliders $\quad 7$

3.2 BP II $\left(\psi \bar{\psi} \rightarrow h_{1} h_{1}\right.$ annihilation channel $)$

$\begin{array}{lll}3.2 .1 & \text { Higgs signal strength } & 9\end{array}$

3.2.2 Triple Higgs coupling 9

3.2.3 Exotic Higgs decays $\quad 9$

3.2.4 Production of $h_{2}$ at colliders 9

3.3 BP III $\left(\psi \bar{\psi} \rightarrow h_{2} h_{2}\right.$ annihilation channel) 9

$\begin{array}{lll}3.3 .1 & \text { Higgs signal strength } & 10\end{array}$

$\begin{array}{lll}3.3 .2 & \text { Triple Higgs coupling } & 10\end{array}$

$\begin{array}{lll}3.3 .3 & \text { Exotic Higgs decays } & 10\end{array}$

3.3.4 Production of $h_{2}$ at colliders 11

$\begin{array}{lll}4 \text { Conclusions } & 11\end{array}$

\section{Introduction}

The existence of dark matter (DM) in the universe is now well established by various observations of the gravitational interactions of the DM and the anisotropy of cosmic microwave background. In order to investigate its feature as a particle, it is required to observe non-gravitational interactions of DM with the standard model (SM) particles. One of such trials is indirect detections of the DM, which probe the signals from the annihilation or the decay of the DM in the current universe. They can contribute to energetic charged particles, photons, and neutrinos which are observable in satellite and terrestrial detectors. In particular, the gamma-ray signals have always drawn attention in the sense that we can identify the location of the sources.

Interestingly, several independent researches have reported an excess of the gammaray emission from the Galactic center (GC) above the expected astrophysical background from the analysis of the Fermi Large Area Telescope (LAT) data [1-11], confirmed later by the experimental group [12]. The excess can be explained by DM annihilations or 
decays although the explanations by unidentified astrophysical sources still remain viable possibilities. In ref. [13], we examined the possibility that the $\mathrm{GeV}$ scale Fermi gammaray excess at the GC can be explained by the DM annihilation in the singlet fermionic dark matter (SFDM) model $[14,15]$. Within the framework, we showed that the DM annihilation into a $b$-quark pair, Higgs pair, and new scalar pair could provide good fits to the Fermi gamma-ray excess data.

The astrophysical observations alone cannot, however, confirm that the excess is originated indeed from the DM due to the unknown populations of other astrophysical sources near the GC. As a complementary approach to support the DM explanation, it is necessary to probe the relevant DM scenarios in collider experiments where background events are relatively well controlled. Following this strategy, we investigate the detection prospects of the SFDM signals explaining the gamma-ray excess in future colliders such as the HighLuminosity Large Hadron Collider (HL-LHC) and the International Linear Collider (ILC) as reference machines of hadron and lepton colliders. ${ }^{1}$

The reference model parameters in ref. [13] are chosen to explain the Fermi gammaray excess with a best fit for each annihilation channel: resonant $b \bar{b}$ production, Higgs pair production, Higgs and a new scalar production when they are almost degenerate in mass, and new scalar pair production. Then, we can proceed analyses with these reference parameters providing definite predictions for collider phenomenology. Note that the scenario, where the SM-like Higgs boson and the new scalar are too degenerate in mass and the couplings of the new scalar to $W W$ and $Z Z$ are highly suppressed, is quite hard to be probed in collider experiments. Thereby we consider the reference parameters of the other three channels, and name them benchmark point (BP) I, II, and III, in order.

This paper is organized as follows. We briefly describe the SFDM model in section 2. The benchmark scenarios for explaining the Fermi gamma-ray excess are introduced and their possible collider signatures in the future collider experiments are discussed in section 3 . Section 4 is devoted to conclusions.

\section{Singlet fermionic dark matter}

In this section, we summarize the key features of the SFDM model used in explaining the gamma-ray excess from the GC [13]. The dark sector is composed of a real scalar field $S$ and a Dirac fermion field $\psi$, both of which are singlet under the SM gauge group.

The Lagrangian for the dark sector is given by the following renormalizable interactions.

$$
\mathcal{L}^{\text {dark }}=\bar{\psi}\left(i \not \partial-m_{\psi_{0}}\right) \psi+\frac{1}{2} \partial_{\mu} S \partial^{\mu} S-g_{S}\left(\cos \theta \bar{\psi} \psi+\sin \theta \bar{\psi} i \gamma^{5} \psi\right) S-V_{S}(S, H),
$$

where the singlet scalar potential is

$$
V_{S}(S, H)=\frac{1}{2} m_{0}^{2} S^{2}+\lambda_{1} H^{\dagger} H S+\lambda_{2} H^{\dagger} H S^{2}+\frac{\lambda_{3}}{3 !} S^{3}+\frac{\lambda_{4}}{4 !} S^{4} .
$$

\footnotetext{
${ }^{1}$ Note that the analysis results in the ILC can be easily converted to those in the Circular Electron Positron Collider (CEPC) as well.
} 
As compared to the original proposal of the SFDM [14], the pseudoscalar interaction in the dark sector is further introduced to obtain a good fit to the gamma-ray excess from the GC using the DM annihilation. See ref. [13] for the detail of the fit to the gamma-ray data.

The SM Higgs potential is given as

$$
V_{\mathrm{SM}}=-\mu^{2} H^{\dagger} H+\lambda_{0}\left(H^{\dagger} H\right)^{2} .
$$

The Higgs doublet $H$ is written in the unitary gauge after the electroweak symmetry breaking as follows:

$$
H=\frac{1}{\sqrt{2}}\left(\begin{array}{c}
0 \\
v_{h}+h
\end{array}\right)
$$

with $v_{h} \simeq 246 \mathrm{GeV}$. The singlet scalar field also develops a nonzero vacuum expectation value, $v_{s}$, and the singlet scalar field is written as $S=v_{s}+s$. The mass parameters $\mu^{2}$ and $m_{0}^{2}$ can be expressed in terms of other parameters by using the minimization condition of the full scalar potential $V_{S}+V_{\mathrm{SM}}$, i.e.,

$$
\begin{aligned}
\mu^{2} & =\lambda_{0} v_{h}^{2}+\left(\lambda_{1}+\lambda_{2} v_{s}\right) v_{s}, \\
m_{0}^{2} & =-\left(\frac{\lambda_{1}}{2 v_{s}}+\lambda_{2}\right) v_{h}^{2}-\left(\frac{\lambda_{3}}{2 v_{s}}+\frac{\lambda_{4}}{6}\right) v_{s}^{2} .
\end{aligned}
$$

The mass terms of the scalar fields are

$$
-\mathcal{L}_{\text {mass }}=\frac{1}{2} \mu_{h}^{2} h^{2}+\frac{1}{2} \mu_{s}^{2} s^{2}+\mu_{h s}^{2} h s,
$$

where

$$
\begin{aligned}
\mu_{h}^{2} & =2 \lambda_{0} v_{h}^{2}, \\
\mu_{s}^{2} & =-\frac{\lambda_{1} v_{h}^{2}}{2 v_{s}}+\frac{\left(3 \lambda_{3}+2 \lambda_{4} v_{s}\right) v_{s}}{6}, \\
\mu_{h s}^{2} & =\left(\lambda_{1}+2 \lambda_{2} v_{s}\right) v_{h} .
\end{aligned}
$$

A non-vanishing value of $\mu_{h s}^{2}$ induces mixing between the SM Higgs field configuration $h$ and the singlet scalar field $s$ as

$$
\left(\begin{array}{l}
h_{1} \\
h_{2}
\end{array}\right)=\left(\begin{array}{cc}
\cos \theta_{s} & \sin \theta_{s} \\
-\sin \theta_{s} & \cos \theta_{s}
\end{array}\right)\left(\begin{array}{l}
h \\
s
\end{array}\right),
$$

The mixing angle $\theta_{s}$ is given by

$$
\tan \theta_{s}=\frac{y}{1+\sqrt{1+y^{2}}}
$$

with $y \equiv 2 \mu_{h s}^{2} /\left(\mu_{h}^{2}-\mu_{s}^{2}\right)$. Then, the physical Higgs boson masses are

$$
m_{h_{1}, h_{2}}^{2}=\frac{1}{2}\left[\left(\mu_{h}^{2}+\mu_{s}^{2}\right) \pm\left(\mu_{h}^{2}-\mu_{s}^{2}\right) \sqrt{1+y^{2}}\right] .
$$


We define that $h_{1}$ is the SM-like Higgs boson with $m_{h_{1}}=125 \mathrm{GeV}$ and $h_{2}$ is the singlet-like scalar boson throughout this paper.

The imaginary mass term of the DM particle $\psi$ from the pseudoscalar interaction, proportional to $\sin \theta$ in (2.1), can be eliminated by a chiral transformation and field redefinition, as stated in refs. $[13,16]$. Then, one can find that the DM mass is given by

$$
m_{\psi}=\sqrt{\left(m_{\psi_{0}}+g_{S} v_{s} \cos \theta\right)^{2}+g_{S}^{2} v_{s}^{2} \sin ^{2} \theta} .
$$

And the dark sector Yukawa interactions are redefined as

$$
-\mathcal{L}_{\text {int }}^{\text {dark }}=g_{S} \cos \xi s \bar{\psi} \psi+g_{S} \sin \xi s \bar{\psi} i \gamma^{5} \psi,
$$

where

$$
\begin{aligned}
\cos \xi & =\frac{m_{\psi_{0}} \cos \theta+g_{S} v_{s}}{m_{\psi}}, \\
\sin \xi & =\frac{m_{\psi_{0}} \sin \theta}{m_{\psi}} .
\end{aligned}
$$

Therefore, there are three independent model parameters for the singlet fermion: $m_{\psi}, g_{S}$, and $\xi$. On the other hand, the masses $m_{h_{1}, h_{2}}$, the mixing angle $\theta_{s}$, and self-couplings of the two physical Higgs particles $h_{1}$ and $h_{2}$ are determined by the six independent parameters in the scalar potential, $\lambda_{0}, \lambda_{1}, \lambda_{2}, \lambda_{3}, \lambda_{4}$, and $v_{s}$. For a reference, we recall that the scalar triple Higgs self-couplings $c_{i j k}$ for $h_{i} h_{j} h_{k}$ interactions are expressed as

$$
\begin{aligned}
c_{111}= & 6 \lambda_{0} v_{h} \cos ^{3} \theta_{s}+\left(3 \lambda_{1}+6 \lambda_{2} v_{s}\right) \cos ^{2} \theta_{s} \sin \theta_{s}+6 \lambda_{2} v_{h} \cos \theta_{s} \sin ^{2} \theta_{s}+\left(\lambda_{3}+\lambda_{4} v_{s}\right) \sin ^{3} \theta_{s}, \\
c_{112}= & -6 \lambda_{0} v_{h} \cos ^{2} \theta_{s} \sin \theta_{s}+2 \lambda_{2} v_{h}\left(2 \cos ^{2} \theta_{s} \sin \theta_{s}-\sin ^{3} \theta_{s}\right) \\
& +\left(\lambda_{1}+2 \lambda_{2} v_{s}\right)\left(\cos ^{3} \theta_{s}-2 \cos \theta_{s} \sin ^{2} \theta_{s}\right)+\left(\lambda_{3}+\lambda_{4} v_{s}\right) \cos \theta_{s} \sin ^{2} \theta_{s}, \\
c_{122}= & 6 \lambda_{0} v_{h} \cos \theta_{s} \sin ^{2} \theta_{s}+2 \lambda_{2} v_{h}\left(\cos ^{3} \theta_{s}-2 \cos \theta_{s} \sin ^{2} \theta_{s}\right) \\
& -\left(\lambda_{1}+2 \lambda_{2} v_{s}\right)\left(2 \cos ^{2} \theta_{s} \sin \theta_{s}-\sin ^{3} \theta_{s}\right)+\left(\lambda_{3}+\lambda_{4} v_{s}\right) \cos ^{2} \theta_{s} \sin \theta_{s}, \\
c_{222}= & -6 \lambda_{0} v_{h} \sin ^{3} \theta_{s}+\left(3 \lambda_{1}+6 \lambda_{2} v_{s}\right) \sin ^{2} \theta_{s} \cos \theta_{s}-6 \lambda_{2} v_{h} \sin \theta_{s} \cos ^{2} \theta_{s} \\
& +\left(\lambda_{3}+\lambda_{4} v_{s}\right) \cos ^{3} \theta_{s} .
\end{aligned}
$$

\section{Benchmark points and collider signatures}

In ref. [13], we demonstrated in detail that several annihilation processes of the DM could provide good fits to the Fermi gamma-ray excess data in the SFDM model. We consider three benchmark points according to the final state of the main DM annihilation process. The first benchmark point corresponds to the DM annihilation channel into a $b$-quark pair (BP I), while the second and third benchmark points correspond to the DM annihilation channels into a SM-like Higgs pair (BP II) and into a new scalar pair (BP III), respectively, which subsequently decay into the SM particles.

The model parameters of each benchmark scenario are fixed by fitting the Fermi gamma-ray excess data, and it gives rise to definite predictions for collider phenomenology 
as will be seen shortly. A benchmark point will be strongly favored as a solution for the Fermi gamma-ray excess if the prediction is confirmed by the future collider experiments. Let us now discuss the details of the three benchmark points and their collider signatures one by one.

\subsection{BP I $(\psi \bar{\psi} \rightarrow b \bar{b}$ annihilation channel $)$}

The channel of DM annihilation into a $b$-quark pair is one of the most widely considered possibilities explaining the gamma-ray excess. For example, the model independent study in ref. [11] showed that the DM annihilation into $b \bar{b}$ gives a good fit for the gamma-ray excess data if $m_{\mathrm{DM}} \simeq 48.7 \mathrm{GeV}$ and the DM annihilation cross section $\langle\sigma v\rangle \simeq 1.75 \times 10^{-26} \mathrm{~cm}^{3} \mathrm{~s}^{-1}$ for a self-conjugate DM. In the SFDM model, this channel can give a best fit to the observed data when $m_{\psi}=49.706 \mathrm{GeV}, m_{h_{2}}=99.416 \mathrm{GeV}, m_{h_{1}}=125.3 \mathrm{GeV}$, and $\sin \theta_{s}=-0.117$ from the reference parameters $\lambda_{0}=0.128816, \lambda_{1}=36.625338 \mathrm{GeV}, \lambda_{2}=-0.131185, \lambda_{3}=$ $-333.447606 \mathrm{GeV}, \lambda_{4}=5.648618, v_{s}=150.017297 \mathrm{GeV}, g_{S}=0.055$, and $\sin \xi=0.01$ [13].

With the parameter setup, we obtain the correct DM relic density $\Omega h^{2}=0.118$, and the DM annihilation cross section $\langle\sigma v\rangle=1.5 \times 10^{-26} \mathrm{~cm}^{3} \mathrm{~s}^{-1}$ which can explain the Fermi gamma-ray excess within the uncertainty of the galaxy halo profile near the GC. Note, however, that only a narrow parameter region around the resonance of $\psi \bar{\psi} \rightarrow b \bar{b}$ is allowed to fit the correct relic density. Furthermore, the new scalar $h_{2}$ needs to be almost scalar in the dark sector, i.e., $\sin \xi=0.01$, to avoid the strong astrophysical bounds from the observation of the gamma-rays coming from the dwarf spheroidal galaxies by Fermi-LAT [17] and the antiproton ratio by PAMELA [18] and AMS-02 [19]. On the one hand, the small mixing angle between the SM-like Higgs and the singlet scalar, i.e., $\sin \theta_{s}=-0.117$ suppresses the spin-independent cross section of the DM recoiling against nucleon as $\sim 6.3 \times 10^{-48} \mathrm{~cm}^{2}$, which is below the constraints from various DM direct detection experiments.

In the following subsections, we discuss the collider signatures of this benchmark point in terms of the Higgs signal strength, triple Higgs coupling, exotic Higgs decays, and direct production of $h_{2}$.

\subsubsection{Higgs signal strength}

As already mentioned, the physical Higgs states are admixtures of $h$ and $s$ in the SFDM model. Therefore, the SM-like Higgs couplings to SM gauge bosons and fermions are universally suppressed by the factor of $\cos \theta_{s}$, compared to the couplings in the SM.

The universal reduction factor $\cos \theta_{s}$ can be precisely measured at the ILC. The SM cross section for the Higgsstrahlung process $e^{+} e^{-} \rightarrow Z h$ reaches its maximum value at $\sqrt{s}=250 \mathrm{GeV}$. About a half million $Z h$ events are expected from the integrated luminosity of $2 \mathrm{ab}^{-1}$ with a suitable polarization of $e^{+} e^{-}$beams. Then, it is possible to precisely measure the inclusive cross section of the Higgsstrahlung process using the recoil mass technique, which disregards the decay products of the Higgs boson. For a $Z$ boson decaying into a pair of fermions, $Z \rightarrow f \bar{f}$, the recoil mass is defined as [20, 21]

$$
M_{\text {recoil }}=\sqrt{s-2 \sqrt{s}\left(E_{f}+E_{\bar{f}}\right)+m_{f \bar{f}}^{2}} .
$$


It corresponds to the mass of the Higgs boson associated with the $Z$ boson in the Higgsstrahlung process. On the other hand, the simplest approach to represent the effect of new physics is the so-called $\kappa$ formalism, where the form of Higgs interactions to the SM particles are the same as the SM, but the couplings are rescaled from the SM value. In the SFDM model, the cross section $\sigma_{Z h_{1}}$ is proportional to $\cos ^{2} \theta_{s}$. The $\cos \theta_{s}$ value can be determined very precisely, with accuracy of $0.38 \%$ assuming a total integrated luminosity of $2 \mathrm{ab}^{-1}$ in the $\kappa$ formalism:

$$
\kappa_{Z}^{2}=\frac{\sigma\left(e^{+} e^{-} \rightarrow Z h_{1}\right)}{\sigma\left(e^{+} e^{-} \rightarrow Z h\right)}=\cos ^{2} \theta_{s},
$$

where the denominator is the SM prediction. Higher energy stages of the ILC experiments will reach an accuracy of $0.3 \%$ for the $h Z Z$ coupling [22]. ${ }^{2}$

The mixing angle $\sin \theta_{s}=-0.117$ of the BP I implies $0.7 \%$ deviation of the $h Z Z$ coupling from the SM value. Therefore the ILC experiment will be able to measure the deviation. On the other hand, the HL-LHC experiment is expected to have an accuracy of $\sim 4 \%[24,25]$, which is insensitive to the deviation of the Higgs coupling.

\subsubsection{Triple Higgs coupling}

Another possible deviation from the SM couplings comes from the triple Higgs self-coupling $c_{111}$. In the SFDM model, the Higgs self-couplings are given by eq. (2.14). The prospects for measuring the Higgs self-coupling is not so promising at the LHC, even at the HL-LHC. Only $\mathcal{O}(1)$ accuracy is expected for the triple Higgs coupling $c_{111}$ from the observation of the Higgs pair production in the channel $h h \rightarrow b \bar{b} \gamma \gamma$ at the HL-LHC [26]. Meanwhile, projections for $100 \mathrm{TeV} p p$ colliders show that a very good precision on the determination of the triple Higgs coupling, a statistical precision of the order of $4 \%$, is achievable using the $b \bar{b} \gamma \gamma$ channel with the integrated luminosity of $30 \mathrm{ab}^{-1}[27,28]$. For the BP I, the triple Higgs coupling $c_{111}$ is $183.95 \mathrm{GeV}$, which corresponds to $3.9 \%$ reduction from the SM value, so it is on the sensitivity limit of the $100 \mathrm{TeV} p p$ collider.

As for the ILC, high-energy machines with center-of-mass energies above $350 \mathrm{GeV}$ can provide the opportunity of directly probing the coupling $c_{111}$ through Higgs-pair production processes, in particular, the double Higgsstrahlung $e^{+} e^{-} \rightarrow Z h h$ and $W W$-fusion $e^{+} e^{-} \rightarrow$ $\nu \bar{\nu} h h$ processes. It is known that the interference between diagrams with and without a triple Higgs vertex has opposite sign in double Higgsstrahlung and $W W$-fusion, so that a combination of double Higgsstrahlung and $W W$-fusion measurements could be used to maximize the precision for the deviation of the triple Higgs coupling. ILC runs at $500 \mathrm{GeV}$ or higher energies maximize the overall precision allowing for a determination of $c_{111}$ with a $\sim 20 \%$ uncertainty at $68 \%$ C.L. [23]

\subsubsection{Exotic Higgs decays}

As mentioned in subsection 3.1.1, the ILC can measure the absolute size of the inclusive Higgs production cross section $\sigma\left(e^{+} e^{-} \rightarrow Z h_{1}\right)$ by applying the recoil mass technique,

\footnotetext{
${ }^{2}$ Furthermore, it is expected that the FCC-ee experiments at $240 \mathrm{GeV}\left(5 \mathrm{ab}^{-1}\right)$ and at $365 \mathrm{GeV}(1.5$ $\left.a b^{-1}\right)$, combined with measurements of single and double Higgs processes at the HL-LHC, can achieve $\sim 0.25 \%$ of the accuracy for $h Z Z$ coupling [23].
} 
which is independent of the Higgs decay modes. The recoil mass technique is applicable even if the Higgs decays invisibly and hence indispensable for extracting the Higgs branching ratio. For the sensitivity to invisible decay modes of the Higgs boson, the $250 \mathrm{GeV}$ ILC with $2 \mathrm{ab}^{-1}$ luminosity and polarized beams would provide an upper limit $\mathrm{BR}(h \rightarrow$ invisible $)<$ $0.3 \%$ at $95 \%$ C.L. [29], which is a factor of 20 below the expected sensitivity of the HLLHC $[24,30]$.

The SFDM model can lead to new Higgs decay channels if kinematically allowed. In the BP I, the mass of the singlet fermion is $m_{\psi} \simeq 50 \mathrm{GeV}$ so that the SM-like Higgs $h_{1}$ can decay into a pair of DM particles, which will escape the detector without leaving tracks, thus leading to an invisible Higgs decay. The branching ratio of the invisible Higgs decay is predicted to be $\operatorname{BR}\left(h_{1} \rightarrow \psi \bar{\psi}\right)=0.73 \%$. Hence, we expect that the invisible Higgs decay of the BP I is within the reach of the ILC, while it is beyond the reach of the HL-LHC.

\subsubsection{Production of $h_{2}$ at colliders}

The new particles can be produced at colliders, thus enabling a direct probe of the SFDM model through dedicated search channels. The search for the light additional Higgs boson $h_{2}\left(m_{h_{2}}<m_{h_{1}}\right)$ directly produced via the gluon-gluon fusion process at hadron colliders suffers from a huge amount of backgrounds if $h_{2} \rightarrow b \bar{b}$ is the dominant decay mode. Nevertheless, the LHC increases the sensitivity on the search for the light Higgs by combining with the other production channels. For instance, the CMS collaboration studied a lowmass resonance in the diphoton channel by combining the $8 \mathrm{TeV}$ and $13 \mathrm{TeV}$ data [31]. ${ }^{3}$ The CMS analysis result sets the upper limit to the ratio of the production cross sections as

$$
\frac{\sigma\left(p p \rightarrow h_{2} \rightarrow \gamma \gamma\right)}{\sigma(p p \rightarrow h \rightarrow \gamma \gamma)_{\mathrm{SM}}}=\sin ^{2} \theta_{s} \lesssim 0.25
$$

at $95 \%$ C.L. for $m_{h_{2}}=m_{h} \simeq 99 \mathrm{GeV}$. For the HL-LHC, we expect that the upper limit will be improved by order of magnitude, $\sin ^{2} \theta_{s} \lesssim \mathcal{O}(0.01)$, so that the production of $h_{2}$ can be marginally discovered.

At the present time, the strongest bound on $\sin ^{2} \theta_{s}$ from the direct production of $h_{2}$ still comes from LEP. The LEP experiments provide the 95\% C.L. upper bound on the Higgs mixing angle $\sin ^{2} \theta_{s}$ as a function of the light Higgs mass $m_{h_{2}}$, which corresponds to $\sin ^{2} \theta_{s} \simeq 0.01$ for $m_{h_{2}}=20 \mathrm{GeV}$ and $\sin ^{2} \theta_{s} \simeq 0.1$ for $m_{h_{2}}=100 \mathrm{GeV}$ [33].

The bounds from the ILC experiment can significantly supersede the LEP bounds due to the higher luminosity by a factor of a thousand as well as polarized beams. The main production processes for light Higgs bosons at the ILC are Higgsstrahlung $\left(e^{+} e^{-} \rightarrow Z h_{2}\right)$ for low center-of-mass energies and $W W$-fusion $\left(e^{+} e^{-} \rightarrow \nu \bar{\nu} h_{2}\right)$ for high center-of-mass energies. By using MadGraph 5 [34], we obtain the production cross sections of $e^{+} e^{-} \rightarrow Z h_{2}$ and $e^{+} e^{-} \rightarrow \nu \bar{\nu} h_{2}$ for polarized beam $P\left(e^{-}, e^{+}\right)=(-80 \%,+30 \%)$. Figure 1 shows the cross sections at various center-of-mass energies.

Here we concentrate on the ILC run at $\sqrt{s}=250 \mathrm{GeV}$ and apply the nominal results in ref. [35] for the $250 \mathrm{GeV}$ with the integrated luminosity $2000 \mathrm{fb}^{-1}$, which are extrapolated

\footnotetext{
${ }^{3}$ See also ref. [32] and the references therein.
} 


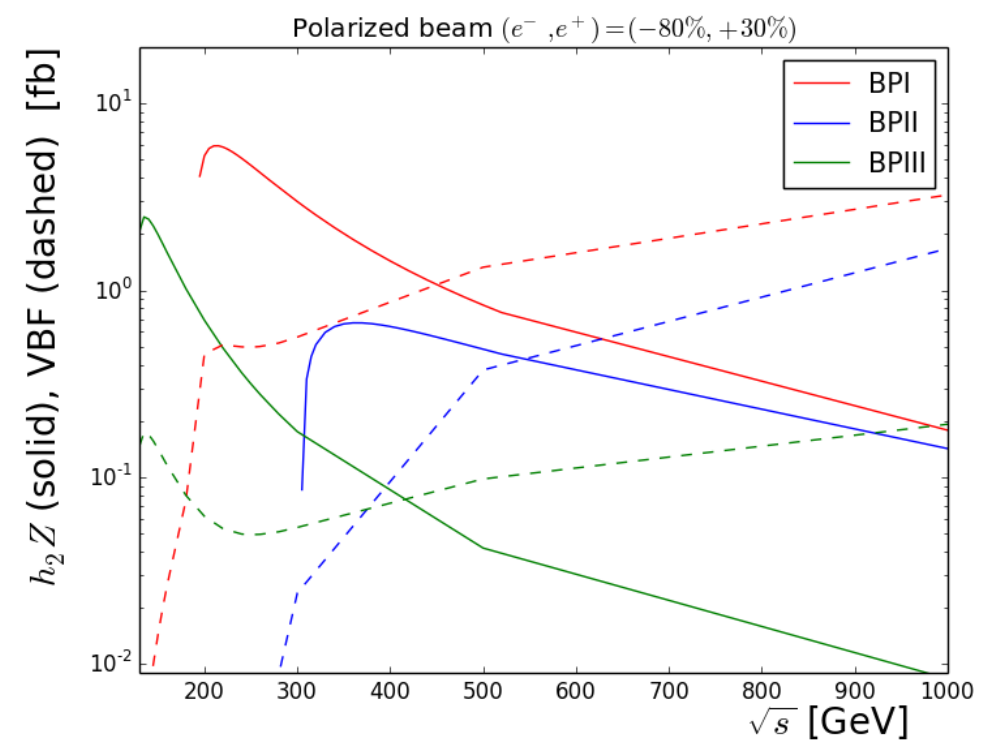

Figure 1. The production cross sections for $h_{2}$ at various $\sqrt{s}$ in $\mathrm{GeV}$ with polarized beams. The solid curves correspond to the Higgsstrahlung process $e^{+} e^{-} \rightarrow Z h_{2}$, and the dashed curves are the $W W$-fusion process $e^{+} e^{-} \rightarrow \nu \bar{\nu} h_{2}$. The red, blue, and green colors denote the BP I, II, and III, respectively.

from the LEP results with the beam polarization $P\left(e^{-}, e^{+}\right)=(-80 \%,+30 \%)$. Two approaches, 'ILC traditional' and 'ILC recoil', are studied for estimating the sensitivity of the ILC to light Higgs masses. In the ILC traditional method, the signal process is identified with light Higgs decay $h_{2} \rightarrow b \bar{b}$ and $Z$ boson decay $Z \rightarrow \mu^{+} \mu^{-}$. On the other hand, in the ILC recoil method, only $Z$ boson decay $\left(Z \rightarrow \mu^{+} \mu^{-}\right)$is exploited and the recoil mass distribution is studied. The $95 \%$ C.L. upper limits on the Higgs mixing angle $\sin ^{2} \theta_{s}$ are obtained for small Higgs masses below $125 \mathrm{GeV}$ as follows [35]:

$$
\begin{array}{ll}
\sin ^{2} \theta_{s} \in[0.001,0.002] & \text { with ILC traditional, } \\
\sin ^{2} \theta_{s} \in[0.003,0.005] & \text { with ILC recoil. }
\end{array}
$$

For $m_{h_{2}}=99.4 \mathrm{GeV}$, the corresponding maximal reach at the ILC is $\sin ^{2} \theta_{s} \simeq 0.0015$. Therefore, the signal of the light Higgs $h_{2}$ can be well discovered in the case of the BP I, where $\sin ^{2} \theta_{s}=0.014$.

\subsection{BP II $\left(\psi \bar{\psi} \rightarrow h_{1} h_{1}\right.$ annihilation channel $)$}

The channel of DM annihilation into the SM-like Higgs pair $\left(h_{1} h_{1}\right.$ in the SFDM) is an alternative possibility explaining the gamma-ray excess. For example, a model independent study in ref. [11] also showed that the DM annihilation into the Higgs pair gives a good fit for the gamma-ray excess data if $m_{\mathrm{DM}} \simeq m_{h_{1}} \simeq 125.7 \mathrm{GeV}$ and the DM annihilation cross section $\langle\sigma v\rangle \simeq 5.33 \times 10^{-26} \mathrm{~cm}^{3} \mathrm{~s}^{-1}$ for a self-conjugate DM.

\footnotetext{
${ }^{4}$ See ref. [36] for a more conservative estimation.
} 
In the SFDM model, this channel gives a best fit when $m_{\psi}=127.5 \mathrm{GeV}, m_{h_{1}}=$ $124.9 \mathrm{GeV}, m_{h_{2}}=213.5 \mathrm{GeV}$, and $\sin \theta_{s}=-0.11$ from the Lagrangian parameters $\lambda_{0}=$ $0.1315, \lambda_{1}=1237.8 \mathrm{GeV}, \lambda_{2}=-2.0, \lambda_{3}=-820.5 \mathrm{GeV}, \lambda_{4}=9.39, v_{s}=306.15 \mathrm{GeV}$, $g_{S}=0.098$, and $\sin \xi=1$ [13]. From theses parameters, we obtain the correct DM relic density $\Omega h^{2}=0.12$, and DM annihilation cross section $\langle\sigma v\rangle=2.11 \times 10^{-26} \mathrm{~cm}^{3} \mathrm{~s}^{-1}$, explaining the gamma-ray excess within the uncertainty of the galaxy halo profile near the GC. Note that the BP II is safe from various astrophysical bounds even when the light Higgs $h_{2}$ is purely pseudoscalar in the dark sector. In the following subsections, we discuss the collider signatures of this benchmark point.

\subsubsection{Higgs signal strength}

In the BP II, the Higgs mixing angle is $\sin \theta_{s}=-0.11$, i.e., $\cos \theta_{s}=0.994$, which implies a $0.6 \%$ deviation of $h Z Z$ coupling from the SM value. As commented in the previous subsection, the deviation is within the reach of the $250 \mathrm{GeV}$ ILC $(0.38 \%)$.

\subsubsection{Triple Higgs coupling}

The triple Higgs coupling $c_{111}=148.96 \mathrm{GeV}$ of the BP II corresponds to $22 \%$ deviation from the SM value. The deviation can be marginally detected at the ILC with higher centerof-mass energy whose accuracy is expected to be $\sim 20 \%$ [23]. On the other hand, it can be well measured in the $100 \mathrm{TeV} p p$ collider where $\sim 4 \%$ accuracy might be reachable $[24,25]$.

\subsubsection{Exotic Higgs decays}

Since $m_{h_{2}}>m_{h_{1}}$ and $2 m_{\psi}>m_{h_{1}}$, exotic Higgs decays such as $h_{1} \rightarrow h_{2} h_{2}$ and $h_{1} \rightarrow \psi \bar{\psi}$ are not expected for the BP II.

\subsubsection{Production of $h_{2}$ at colliders}

The singlet-like Higgs mass of the BP II is $m_{h_{2}}=213.5 \mathrm{GeV}$. For the mass region of $m_{h_{1}}<m_{h_{2}}<2 m_{h_{1}}, h_{2}$ dominantly decays to the pairs of $W W$ and $Z Z$. The corresponding branching ratios are $\mathrm{BR}\left(h_{2} \rightarrow W W\right)=71.5 \%$ and $\mathrm{BR}\left(h_{2} \rightarrow Z Z\right)=28.0 \%$ and the total decay width of the $h_{2}$ is about $25 \mathrm{MeV}$. The strongest constraint for the additional Higgs in this mass range comes from the current LHC searches for a new scalar resonance decaying to a pair of $Z$ bosons [37-39]. The 95\% C.L. upper bound on $\sigma(p p \rightarrow X \rightarrow Z Z)$ at the LHC with an integrated luminosity of $\sim 36 \mathrm{fb}^{-1}$ at the center-of-mass energy of $13 \mathrm{TeV}$, is about $2.8 \times 10^{-1} \mathrm{pb}$ for $m_{X} \simeq 210 \mathrm{GeV}$. This can be translated to the upper bound on the Higgs mixing angle as $\sin ^{2} \theta_{s} \lesssim 0.06$ for $m_{H} \simeq 210 \mathrm{GeV}$. At the HL-LHC with the integrated luminosity of $3000 \mathrm{fb}^{-1}$, an order of magnitude improvement on the bound, $\sin ^{2} \theta_{s} \lesssim 0.008$ at $95 \%$ C.L., is expected [40]. Therefore, we expect that the corresponding signal of $h_{2}$ with the mixing angle $\sin ^{2} \theta_{s}=0.012$ can be observed at the HL-LHC.

\subsection{BP III ( $\psi \bar{\psi} \rightarrow h_{2} h_{2}$ annihilation channel $)$}

Another viable scenario for the Fermi gamma-ray excess is the DM annihilation process into dark sector particles. The scenario is the most promising channel explaining the gammaray excess in the sense that it is rather easy to avoid various bounds from colliders and 
astrophysical observations. The model independent study in ref. [41] shows that the DM annihilation into a pair of new scalars $(\phi \phi)$, each of which decays to a $b$-quark pair, can provide a good fit for the Fermi gamma-ray excess data. The best fit is obtained for $m_{\mathrm{DM}} \simeq$ $65 \mathrm{GeV}$ and the new scalar mass is about the half of the DM mass $m_{\phi}=m_{\mathrm{DM}} / 2$. The DM annihilation cross section is $\langle\sigma v\rangle \simeq 2.45 \times 10^{-26} \mathrm{~cm}^{3} \mathrm{~s}^{-1}$, assuming a self-conjugate DM.

In the SFDM, the best fit to the excess is obtained when $m_{\psi}=69.2 \mathrm{GeV}, m_{h_{1}}=$ $125.1 \mathrm{GeV}, m_{h_{2}}=35.7 \mathrm{GeV}$ and $\sin \theta_{s}=0.025$ from the parameters in the Lagrangian $\lambda_{0}=0.13, \lambda_{1}=4.5 \mathrm{GeV}, \lambda_{2}=-0.0055, \lambda_{3}=-391.51 \mathrm{GeV}, \lambda_{4}=2.20, v_{s}=276.21 \mathrm{GeV}$, and $g_{S}=0.056$. With these parameter setup, we obtain the correct DM relic density $\Omega h^{2}=0.121$, and the DM annihilation cross section $\langle\sigma v\rangle=2.26 \times 10^{-26} \mathrm{~cm}^{3} \mathrm{~s}^{-1}$. The benchmark scenario can explain the Fermi gamma-ray excess within the uncertainty of the galaxy halo profile near the GC like the other benchmark points. We discuss the collider signatures of the BP III in this subsection.

\subsubsection{Higgs signal strength}

The Higgs mixing angle $\sin \theta_{s}=0.025$ corresponds to $\cos \theta_{s}=0.9997$. It implies $0.03 \%$ reduction of $h Z Z$ coupling from the SM value. The future colliders including the ILC are not sensitive enough to measure such a small deviation.

\subsubsection{Triple Higgs coupling}

The triple Higgs coupling of the BP III is $c_{111}=190.57 \mathrm{GeV}$, which implies $-0.15 \%$ deviation from the SM prediction. Such a small deviation will be very difficult to measure even in the $100 \mathrm{TeV} p p$ collider.

\subsubsection{Exotic Higgs decays}

The singlet-like Higgs mass of the BP III $\left(m_{h_{2}}=35.7 \mathrm{GeV}\right)$ is smaller than half of the SM-like Higgs mass, thus $h_{1}$ can decay into a new scalar pair $h_{2} h_{2}$. The corresponding branching ratio is $\operatorname{BR}\left(h_{1} \rightarrow h_{2} h_{2}\right)=7 \%$. The light Higgs subsequently decays to mostly $b \bar{b}$, with $\operatorname{BR}\left(h_{2} \rightarrow b \bar{b}\right)=86 \%$, giving rise to $\operatorname{BR}\left(h_{1} \rightarrow h_{2} h_{2} \rightarrow 4 b\right)=5.2 \%$.

Recently, the ATLAS collaboration has provided a result of search for the Higgs boson produced in association with a vector boson and decaying into two spin-zero particles in the $H \rightarrow a a \rightarrow 4 b$ channel at the $13 \mathrm{TeV}$ LHC with an integrated luminosity of $36.1 \mathrm{fb}^{-1}$ [42]. The $95 \%$ C.L. upper limit on the combination of cross sections for $W H$ and $Z H$ times the branching ratio of $H \rightarrow a a \rightarrow 4 b$ ranges from $3.0 \mathrm{pb}$ for $m_{a}=20 \mathrm{GeV}$ to $1.3 \mathrm{pb}$ for $m_{a}=60 \mathrm{GeV}$. The upper limit is about $1.1 \mathrm{pb}$ for $m_{a}=35.7 \mathrm{GeV}$. We can translate the result to an upper limit on the branching ratio $\mathrm{BR}\left(h_{1} \rightarrow h_{2} h_{2} \rightarrow 4 b\right) \lesssim 50 \%$ with $m_{h_{2}}=35.7 \mathrm{GeV}$ for the BP III, assuming the SM cross sections for $W H$ and $Z H$. An order of improvement on the bound will be possible at the HL-LHC [43].

Searches for exotic Higgs decays with final states involving quarks are somewhat challenging at the LHC and HL-LHC. However, the ILC at $\sqrt{s}=250 \mathrm{GeV}$ has an excellent sensitivity to search for such final states, due to low QCD backgrounds and the recoil mass technique for tagging the Higgs boson associated with the $Z$ boson. Through the Higgsstrahlung process $e^{+} e^{-} \rightarrow Z h_{1}$ and the $h_{1} \rightarrow h_{2} h_{2} \rightarrow 4 b$ decay, the $250 \mathrm{GeV}$ ILC with 


\begin{tabular}{|c|c|c|c|c|}
\hline & Higgs signal strength & Triple Higgs coupling & Exotic Higgs decay & $h_{2}$ production \\
\hline BP I & $\begin{array}{l}0.7 \% \text { reduction } \\
\quad \bigcirc \\
(\delta \sim 0.3 \%(\mathrm{ILC}))\end{array}$ & $\begin{array}{l}3.9 \% \text { reduction } \\
\quad \triangle \\
(\delta \sim 4 \%(\text { FCC-hh }))\end{array}$ & $\begin{array}{c}\mathrm{BR}\left(h_{1} \rightarrow \psi \bar{\psi}\right)=0.73 \% \\
\bigcirc \\
(\mathrm{BR}(h \rightarrow \text { invisible }) \lesssim \\
0.3 \%(\mathrm{ILC}))\end{array}$ & $\begin{array}{c}m_{h_{2}}=99.4 \mathrm{GeV} \\
\sin ^{2} \theta_{s}=0.014 \\
\bigcirc \\
\left(\sin ^{2} \theta_{s} \lesssim 0.0015(\mathrm{ILC})\right)\end{array}$ \\
\hline BP II & $\begin{array}{l}0.6 \% \text { reduction } \\
\quad \bigcirc \\
(\delta \sim 0.3 \%(\mathrm{ILC}))\end{array}$ & $\begin{array}{l}22 \% \text { reduction } \\
\bigcirc \\
(\delta \sim 4 \%(\text { FCC-hh }))\end{array}$ & $\begin{array}{c}\text { No exotic decay } \\
\times\end{array}$ & $\begin{array}{c}m_{h_{2}}=213.5 \mathrm{GeV} \\
\sin ^{2} \theta_{s}=0.012 \\
\bigcirc \\
\left(\sin ^{2} \theta_{s} \lesssim 0.008(\mathrm{HL}-\mathrm{LHC})\right)\end{array}$ \\
\hline BP III & $\begin{array}{l}0.03 \% \text { reduction } \\
\quad \times \\
(\delta \sim 0.3 \%(\mathrm{ILC}))\end{array}$ & $\begin{array}{l}0.15 \% \text { reduction } \\
\quad \times \\
(\delta \sim 4 \%(\text { FCC-hh }))\end{array}$ & $\begin{array}{c}\mathrm{BR}\left(h_{1} \rightarrow h_{2} h_{2}\right)=7 \% \\
\bigcirc \\
(\mathrm{BR}(h \rightarrow s s \rightarrow 4 b) \lesssim \\
0.1 \%(\mathrm{ILC}))\end{array}$ & $\begin{array}{c}m_{h_{2}}=35.7 \mathrm{GeV} \\
\sin ^{2} \theta_{s}=6.3 \times 10^{-4} \\
\times \\
\left(\sin ^{2} \theta_{s} \lesssim 0.0015(\mathrm{ILC})\right)\end{array}$ \\
\hline
\end{tabular}

Table 1. Summary of the collider signatures for the benchmark points. The circle (triangle) denotes that the collider signal of the benchmark can be (marginally) measurable at future colliders. The cases where the collider signal is expected to be hard to measure or beyond the reach of the future colliders are marked with the cross. The texts in parentheses are relevant bounds expected at the future colliders.

the integrated luminosity of $2 \mathrm{ab}^{-1}$ can exclude branching ratio of $h_{1} \rightarrow h_{2} h_{2} \rightarrow 4 b$ down to $\sim 10^{-3}$ [44]. Therefore, we expect that the exotic Higgs decay of the BP III will be very well detected at the ILC experiment.

\subsubsection{Production of $h_{2}$ at colliders}

In the BP III, the Higgs mixing angle is $\sin ^{2} \theta_{s}=6.25 \times 10^{-4}$ and the mass of the singletlike Higgs is $m_{h_{2}}=35.7 \mathrm{GeV}$. Since the mixing angle value is much smaller than the ILC sensitivity given in eq. (3.4), the signal for the $e^{+} e^{-} \rightarrow Z h_{2}$ process is beyond the reach of the ILC experiment.

\section{Conclusions}

In this paper, we investigated various collider signatures of some benchmark points in the SFDM model. The benchmark points were classified according to the final state of the dominant DM annihilation process, and we showed in the previous study that each of them provides a best fit to the gamma-ray excess observed at the GC. Specifically, we chose three benchmark points where the DM annihilates dominantly into a pair of $b$ quarks, SM-like Higgs bosons, and new scalars, dubbed as BP I, BP II, and BP III, respectively. Provided that the SFDM is a proper reference model for the DM, probing their collider signatures is necessary in order to either support or oppose the possibilities of explaining the gamma-ray excess in terms of the DM annihilation.

In the SFDM model, all the particles in the dark sector are singlets, but they are connected with the SM sector through a mixing between the singlet scalar and the Higgs boson. Thus, collider observables related to the Higgs boson serve essential means to test the model. We categorize the search strategies by listing four observables: Higgs signal strength, triple Higgs coupling, exotic Higgs decay, and production of the new scalar, $h_{2}$, at future colliders such as ILC, HL-LHC, and FCC-hh. 
Table 1 summarizes preferred observables for probing each benchmark point. We find that the BP I can be explored by the measurements of the Higgs signal strength, exotic Higgs decay, and $h_{2}$ production at the ILC. On the other hand, as the triple Higgs coupling of the BP I is on the sensitivity limit of the FCC-hh, we expect that observing a deviation of the triple Higgs coupling from the SM prediction will be marginally possible. In the case of the BP II, all the collider observables except the exotic Higgs decay are useful. In particular, the triple Higgs coupling of the BP II is substantially smaller than the SM prediction, so it is easily testable in the future experiments. The BP III can be probed by the search for the exotic Higgs decay $h_{1} \rightarrow h_{2} h_{2}$, while the direct production of $h_{2}$ is hardly measurable due to the tiny mixing angle. Our study shows that combined searches must be performed to find the new physics signals at future lepton and hadron colliders since single collider observable is not sufficient to probe all the benchmark scenarios simultaneously.

\section{Acknowledgments}

We would like to thank Jia Liu, Zhen Liu, and Carlos Wagner for useful discussions. This work was performed in part at the Aspen Center for Physics, which is supported by National Science Foundation grant PHY-1607611. YGK is supported by the Basic Science Research Program through the National Research Foundation of Korea (NRF) funded by the Korean Ministry of Education, Science and Technology (NRF-2018R1D1A1B07050701). The work of CBP is supported by IBS under the project code, IBS-R018-D1. SS is supported by the National Research Foundation of Korea (NRF-2017R1D1A1B03032076). SS appreciates the hospitality of Fermi National Accelerator Laboratory.

Open Access. This article is distributed under the terms of the Creative Commons Attribution License (CC-BY 4.0), which permits any use, distribution and reproduction in any medium, provided the original author(s) and source are credited.

\section{References}

[1] L. Goodenough and D. Hooper, Possible evidence for dark matter annihilation in the inner Milky Way from the Fermi gamma ray space telescope, arXiv:0910.2998 [INSPIRE].

[2] D. Hooper and L. Goodenough, Dark matter annihilation in the galactic center as seen by the Fermi gamma ray space telescope, Phys. Lett. B 697 (2011) 412 [arXiv:1010.2752] [INSPIRE].

[3] D. Hooper and T. Linden, On the origin of the gamma rays from the galactic center, Phys. Rev. D 84 (2011) 123005 [arXiv: 1110.0006] [INSPIRE].

[4] K.N. Abazajian and M. Kaplinghat, Detection of a gamma-ray source in the galactic center consistent with extended emission from dark matter annihilation and concentrated astrophysical emission, Phys. Rev. D 86 (2012) 083511 [Erratum ibid. D 87 (2013) 129902] [arXiv: 1207.6047 ] [INSPIRE].

[5] D. Hooper and T.R. Slatyer, Two emission mechanisms in the Fermi bubbles: a possible signal of annihilating dark matter, Phys. Dark Univ. 2 (2013) 118 [arXiv:1302.6589] [INSPIRE]. 
[6] C. Gordon and O. Macias, Dark matter and pulsar model constraints from galactic center Fermi-LAT gamma ray observations, Phys. Rev. D 88 (2013) 083521 [Erratum ibid. D 89 (2014) 049901] [arXiv: 1306.5725] [INSPIRE].

[7] W.-C. Huang, A. Urbano and W. Xue, Fermi bubbles under dark matter scrutiny. Part I: astrophysical analysis, arXiv:1307.6862 [INSPIRE].

[8] K.N. Abazajian, N. Canac, S. Horiuchi and M. Kaplinghat, Astrophysical and dark matter interpretations of extended gamma-ray emission from the galactic center, Phys. Rev. D 90 (2014) 023526 [arXiv: 1402.4090] [INSPIRE].

[9] T. Daylan et al., The characterization of the gamma-ray signal from the central Milky Way: a case for annihilating dark matter, Phys. Dark Univ. 12 (2016) 1 [arXiv:1402.6703] [INSPIRE].

[10] F. Calore, I. Cholis and C. Weniger, Background model systematics for the Fermi GeV excess, JCAP 03 (2015) 038 [arXiv: 1409.0042] [INSPIRE].

[11] F. Calore, I. Cholis, C. McCabe and C. Weniger, A tale of tails: dark matter interpretations of the Fermi GeV excess in light of background model systematics, Phys. Rev. D 91 (2015) 063003 [arXiv: 1411.4647] [INSPIRE].

[12] Fermi-LAT collaboration, M. Ajello et al., Fermi-LAT observations of high-energy $\gamma$-ray emission toward the galactic center, Astrophys. J. 819 (2016) 44 [arXiv:1511.02938] [INSPIRE].

[13] Y.G. Kim, K.Y. Lee, C.B. Park and S. Shin, Secluded singlet fermionic dark matter driven by the Fermi gamma-ray excess, Phys. Rev. D 93 (2016) 075023 [arXiv:1601.05089] [InSPIRE].

[14] Y.G. Kim, K.Y. Lee and S. Shin, Singlet fermionic dark matter, JHEP 05 (2008) 100 [arXiv:0803.2932] [INSPIRE].

[15] Y.G. Kim and S. Shin, Singlet fermionic dark matter explains DAMA signal, JHEP 05 (2009) 036 [arXiv: 0901.2609] [INSPIRE].

[16] M.A. Fedderke, J.-Y. Chen, E.W. Kolb and L.-T. Wang, The fermionic dark matter Higgs portal: an effective field theory approach, JHEP 08 (2014) 122 [arXiv:1404.2283] [INSPIRE].

[17] Fermi-LAT collaboration, M. Ackermann et al., Searching for dark matter annihilation from Milky Way dwarf spheroidal galaxies with six years of Fermi Large Area Telescope data, Phys. Rev. Lett. 115 (2015) 231301 [arXiv:1503.02641] [INSPIRE].

[18] PAMELA collaboration, O. Adriani et al., PAMELA results on the cosmic-ray antiproton flux from $60 \mathrm{MeV}$ to $180 \mathrm{GeV}$ in kinetic energy, Phys. Rev. Lett. 105 (2010) 121101 [arXiv: 1007.0821] [INSPIRE].

[19] AMS collaboration, M. Aguilar et al., Antiproton flux, antiproton-to-proton flux ratio and properties of elementary particle fluxes in primary cosmic rays measured with the Alpha Magnetic Spectrometer on the International Space Station, Phys. Rev. Lett. 117 (2016) 091103 [INSPIRE].

[20] K. Fujii et al., Physics case for the International Linear Collider, arXiv:1506.05992 [INSPIRE].

[21] J. Liu, X.-P. Wang and F. Yu, A tale of two portals: testing light, hidden new physics at future $e^{+} e^{-}$colliders, JHEP 06 (2017) 077 [arXiv:1704.00730] [INSPIRE]. 
[22] K. Fujii et al., Physics case for the $250 \mathrm{GeV}$ stage of the International Linear Collider, arXiv:1710.07621 [INSPIRE].

[23] S. Di Vita et al., A global view on the Higgs self-coupling at lepton colliders, JHEP 02 (2018) 178 [arXiv: 1711.03978] [INSPIRE].

[24] CMS collaboration, Projected performance of an upgraded CMS detector at the LHC and HL-LHC: contribution to the Snowmass process, in Proceedings, 2013 Community Summer Study on the Future of U.S. Particle Physics: Snowmass on the Mississippi (CSS2013), Minneapolis, MN, U.S.A., 29 July-6 August 2013 [arXiv:1307.7135] [INSPIRE].

[25] ATLAS collaboration, Projections for measurements of Higgs boson signal strengths and coupling parameters with the ATLAS detector at a HL-LHC, ATL-PHYS-PUB-2014-016, CERN, Geneva, Switzerland, (2014).

[26] ATLAS collaboration, Study of the double Higgs production channel $H(\rightarrow b \bar{b}) H(\rightarrow \gamma \gamma)$ with the ATLAS experiment at the HL-LHC, ATL-PHYS-PUB-2017-001, CERN, Geneva, Switzerland, (2017).

[27] H.-J. He, J. Ren and W. Yao, Probing new physics of cubic Higgs boson interaction via Higgs pair production at hadron colliders, Phys. Rev. D 93 (2016) 015003 [arXiv:1506.03302] [INSPIRE].

[28] R. Contino et al., Physics at a $100 \mathrm{TeV}$ pp collider: Higgs and EW symmetry breaking studies, CERN Yellow Report (2017) 255 [arXiv: 1606.09408] [INSPIRE].

[29] T. Barklow et al., Improved formalism for precision Higgs coupling fits, Phys. Rev. D 97 (2018) 053003 [arXiv:1708.08912] [INSPIRE].

[30] ATLAS collaboration, Projections for measurements of Higgs boson cross sections, branching ratios and coupling parameters with the ATLAS detector at a HL-LHC, ATL-PHYS-PUB-2013-014, CERN, Geneva, Switzerland, (2013).

[31] CMS collaboration, Search for new resonances in the diphoton final state in the mass range between 70 and $110 \mathrm{GeV}$ in pp collisions at $\sqrt{s}=8$ and $13 \mathrm{TeV}$, CMS-PAS-HIG-17-013, CERN, Geneva, Switzerland, (2017).

[32] D. Liu, J. Liu, C.E.M. Wagner and X.-P. Wang, A light Higgs at the LHC and the B-anomalies, JHEP 06 (2018) 150 [arXiv: 1805.01476] [INSPIRE].

[33] OPAL, DELPHI, LEP Working Group For Higgs Boson SEARChes, ALEPH and L3 collaborations, R. Barate et al., Search for the Standard Model Higgs boson at LEP, Phys. Lett. B 565 (2003) 61 [hep-ex/0306033] [INSPIRE].

[34] J. Alwall et al., The automated computation of tree-level and next-to-leading order differential cross sections and their matching to parton shower simulations, JHEP 07 (2014) 079 [arXiv: 1405.0301] [INSPIRE].

[35] P. Drechsel, G. Moortgat-Pick and G. Weiglein, Sensitivity of the ILC to light Higgs masses, in International Workshop on Future Linear Collider (LCWS2017), Strasbourg, France, 23-27 October 2017 [arXiv: 1801.09662] [INSPIRE].

[36] Y. Wang, J. List and M. Berggren, Search for light scalars produced in association with muon pairs for $\sqrt{s}=250 \mathrm{GeV}$ at the ILC, in International Workshop on Future Linear Collider (LCWS2017), Strasbourg, France, 23-27 October 2017 [arXiv: 1801.08164] [INSPIRE].

[37] CMS collaboration, Search for a Higgs boson in the mass range from 145 to $1000 \mathrm{GeV}$ decaying to a pair of $W$ or $Z$ bosons, JHEP 10 (2015) 144 [arXiv:1504.00936] [INSPIRE]. 
[38] ATLAS collaboration, Search for heavy $Z Z$ resonances in the $\ell^{+} \ell^{-} \ell^{+} \ell^{-}$and $\ell^{+} \ell^{-} \nu \bar{\nu}$ final states using proton-proton collisions at $\sqrt{s}=13 \mathrm{TeV}$ with the ATLAS detector, Eur. Phys. J. C 78 (2018) 293 [arXiv:1712.06386] [INSPIRE].

[39] CMS collaboration, Search for a new scalar resonance decaying to a pair of $Z$ bosons in proton-proton collisions at $\sqrt{s}=13 \mathrm{TeV}$, JHEP 06 (2018) 127 [arXiv: 1804.01939] [INSPIRE].

[40] CMS collaboration, 2HDM neutral Higgs future analysis studies, CMS-PAS-FTR-13-024, CERN, Geneva, Switzerland, (2013).

[41] B. Dutta, Y. Gao, T. Ghosh and L.E. Strigari, Confronting galactic center and dwarf spheroidal gamma-ray observations with cascade annihilation models, Phys. Rev. D 92 (2015) 075019 [arXiv: 1508.05989] [INSPIRE].

[42] ATLAS collaboration, Search for the Higgs boson produced in association with a vector boson and decaying into two spin-zero particles in the $H \rightarrow a a \rightarrow 4 b$ channel in pp collisions at $\sqrt{s}=13 \mathrm{TeV}$ with the ATLAS detector, JHEP 10 (2018) 031 [arXiv:1806. 07355] [INSPIRE].

[43] D. Curtin et al., Exotic decays of the 125 GeV Higgs boson, Phys. Rev. D 90 (2014) 075004 [arXiv:1312.4992] [INSPIRE].

[44] Z. Liu, L.-T. Wang and H. Zhang, Exotic decays of the $125 \mathrm{GeV}$ Higgs boson at future $e^{+} e^{-}$ lepton colliders, Chin. Phys. C 41 (2017) 063102 [arXiv:1612.09284] [INSPIRE]. 